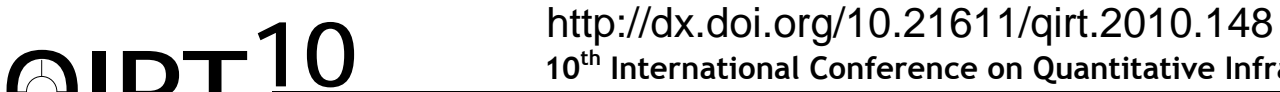 \\ $10^{\text {th }}$ International Conference on Quantitative InfraRed Thermography \\ July 27-30, 2010, Québec (Canada)
}

\section{Optical characterization of growing thin films at high temperatures by analysis of near infrared emissivity variations using CCD thermal imaging}

by G. Zauner, F. Mayrhofer and G. Hendorfer

Upper Austria Univ. of Appl. Sciences - Wels Campus, Stelzhamerstr. 23, 4600 Wels, Austria, gerald.zauner@fh-wels.at

\begin{abstract}
Due to interference effects which occur during the growth of surface thin films, the resulting spectral emissivity varies continuously. It is shown in this paper, that these temporal variations of emissivity allow the determination of the complex index of refraction (i.e. optical constants $n, k$ ) of a thin absorbing film, without explicit knowledge of the optical constants of the substrate layer. In particular, this work presents results obtained by the analysis of the thermal radiation of a thin thermally grown iron-oxide film on a heated steel specimen (at approx. $400^{\circ} \mathrm{C}$ ) measured by a standard CCD camera with a near infrared band-pass filter $(\sim 1 \mu \mathrm{m})$.
\end{abstract}

\section{Introduction}

In this work we present a generally applicable method for determining the optical constants $(n, k)$ of a semitransparent surface thin film, based solely on four measured emissivity values during film growth (i.e. no reflection setup, e.g. as in ellipsometry, is necessary). From this a quantitative and spatio-temporal analysis (e.g. of film-thickness growth) becomes possible. We use a standard CCD camera as thermal imaging device, as the spectral sensitivity of a Si-CCD at $1 \mu \mathrm{m}$ allows the imaging of thermal radiation at temperatures above approx. $400{ }^{\circ} \mathrm{C}$ [1]. Mainly two different approaches for emissivity modeling of thin films can be found in literature. One considers the thermal radiation of the substrate material which passes through the film layer (direct method [2][3]) and the second is based on a reflectance model of the film surface [4]. Our work is based on the latter which assumes the validity of the fundamental equation $\varepsilon=1-\rho$ (even for thin film systems). This assumption was validated by simultaneous measurements of near infrared reflection and emission spectra. Under the assumption of constant optical properties during the film growth of the bulk material and the semitransparent surface film, it is possible to derive a physical model by which variations of the resulting emissivity can be described by variations of film thickness. From this model, which is based on the calculation of the reflectance of a thin absorbing film on an absorbing substrate, the complex index of refraction of the film can be determined without explicit knowledge of the optical constants of the substrate layer. The minima and maxima of the emissivity interference signal and the final constant emissivity value are the only input parameters required for the proposed method. These quantities are easily deduced from a measurement procedure described in chapter 4.

\section{Theory}

\section{Basics of Refraction}

Reflected and transmitted amplitudes and intensities of incident electromagnetic waves are determined by Maxwell's equations. The solution at the boundary layer of different transparent isotropic media leads to the Fresnel reflection and transmission coefficients $r_{p}$ and $t_{s}$. In the case of normal incidence the Fresnel coefficients are given by:

$$
\frac{E_{R p}}{E_{I p}}=r_{p}=-r_{s}=\frac{n_{1}-n_{0}}{n_{0}+n_{1}} ; \quad \frac{E_{T p}}{E_{I p}}=t_{s}=t_{p}=\frac{2 n_{0}}{n_{0}+n_{1}}
$$

where $\mathrm{E}$ denotes the electrical field vector and the subscripts $I, R$, and $T$ indicate incident, reflected and transmitted waves respectively. The direction of the plane of polarization is given by the subscript $s$ and $p$ for perpendicular ('senkrecht') and parallel to the plane of incidence. The numerical subscript of the index of refraction $n$ denotes the according medium. The reflectance $\rho$ (which is defined as the ratio of reflected and incident energies) can be obtained from the reflection coefficients by the equation $\rho=|r|^{2}$.In the case of absorbing materials, the index of refraction $n$ is replaced by a complex value $\tilde{n}=n$-ik (where $k$ denotes the extinction coefficient). 


\section{Reflectance and emissivity of a thin film}

The reflectance (and emissivity) of a thin film on a substrate material changes considerably with film thickness [5]. An ideal reflectance model for such a thin film system is shown in Fig.1 [6]:

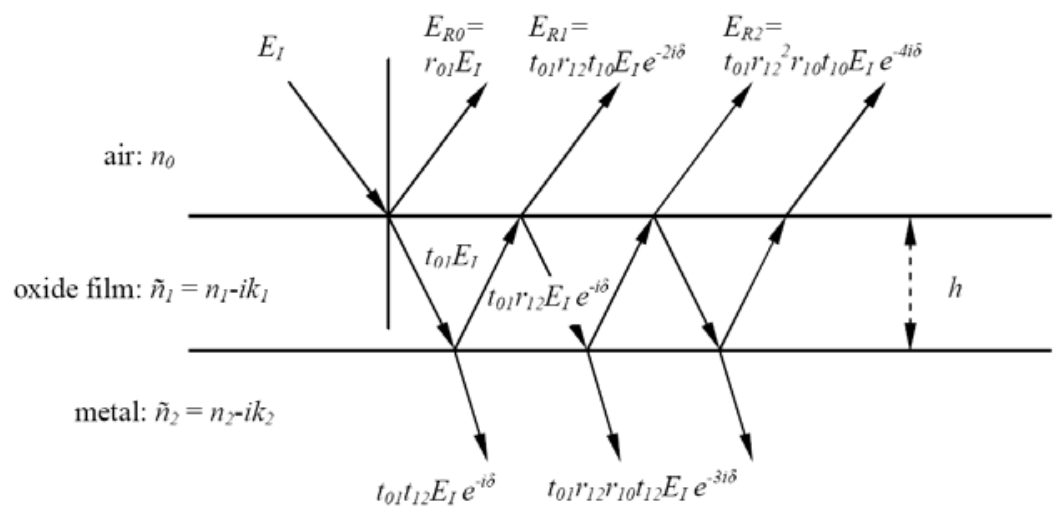

Fig.1: Reflection model: oxide film on a metal substrate.

The change in phase $\delta$ of an electromagnetic wave passing through the film can be calculated by:

$$
\delta=\frac{2 \pi}{\lambda} \tilde{n}_{1} h
$$

where $\lambda$ is the wavelength of the monochromatic incident light and $h$ is the film thickness. The resulting reflected beam in medium 0 is given by:

$$
r=r_{01}+t_{01} t_{10} r_{12} e^{-2 i \delta}+t_{01} t_{10} r_{10} r_{12}{ }^{2} e^{-4 i \delta}+t_{01} t_{10} r_{10}{ }^{2} r_{12} 3 e^{-6 i \delta}+\ldots=r_{01}+\frac{t_{01} t_{10} r_{12} e^{-2 i \delta}}{1+r_{01} r_{12} e^{-2 i \delta}}=\frac{r_{01}+r_{12} e^{-2 i \delta}}{1+r_{01} r_{12} e^{-2 i \delta}}
$$

The (measurable) reflectance of normal incident and non-polarized light can finally be written as:

$$
\rho=\left|r^{2}\right|=\left|r_{01}+\frac{t_{01} t_{10} r_{12} e^{-2 i \delta}}{1+r_{01} r_{12} e^{-2 i \delta}}\right|^{2}=\left|\frac{r_{01}+r_{12} e^{-2 i \delta}}{1+r_{01} r_{12} e^{-2 i \delta}}\right|^{2}
$$

From the law of conservation of energy it follows that $\alpha+\rho+\tau=1$ (where $\alpha, \rho$ and $\tau$ denote absorbance, reflectance and transmission). Taking into account Kirchhoff's law of radiation $(\alpha=\varepsilon)$ and assuming that the transmission can be neglected for thick metallic substrates $(\tau=0)$ the emissivity can be expressed as $\varepsilon=1-\rho$. With the aid of this model the reflectivity (and therefore the emissivity) can be computed if the optical properties of the substrate and film are known (see Fig.2, left, solid line). For further considerations, the exact expression of the right hand side of equation (3) is not used, but only a two-beam interference approximation given by the first two terms of the left hand side. The resulting difference (Fig.2 right, bottom) is shown exemplarily on a representative system assuming a medium 0 as air $\left(n_{0}=1\right)$, medium 1 as an oxide film $\left(\tilde{n}_{1}=2-j 0.5\right)$ and medium 2 as steel $\left(\tilde{n}_{2}=3-j 5\right)$. As Fig.2 (right) shows, the difference between the exact solution and the approximation decreases rapidly with increasing film-thickness. If the film absorption is relatively high (which is in general valid for oxide films) this error can be neglected in film thickness above approx. 80nm.

\section{Envelope of the interference signal}

We focus our analysis on the extreme values of the time varying emissivity signal (maxima and minima). At these points the phase is well defined and therefore it can be expressed by a simplified version of equation (3) - the envelope. The envelope of the emissivity signal (Fig.2 left, dotted lines) can be calculated without taking into account the changes of phase 
of the reflected beams because constructive and destructive interference appears only if this change is a multiple of $2 \pi$ and $\pi$ respectively.
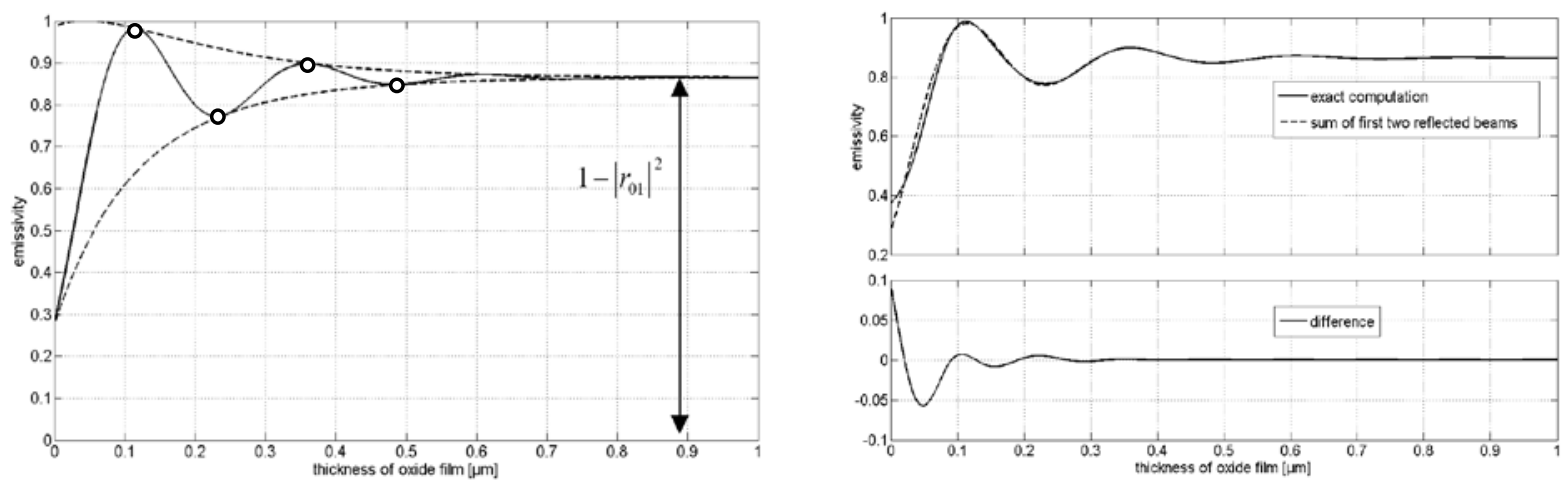

Fig.2: Theoretical results; left: emissivity signal as film thickness increases; right, top: emissivity versus filmthickness (solid line: the exact computation; dotted line: the two-beam approximation (i.e. the sum of the first two reflected beams)); right, bottom: resulting difference.

For this reason, the amplitudes of the first two reflected beams $E_{R 0}$ and $E_{R 1}$ (see Fig.1) have to be determined and then simply added or subtracted. For the second reflected beam the attenuation due to the absorbing film has to be considered. The absorption coefficient $K$ describes the absorbed energy in a medium in terms of its thickness and can be obtained from the imaginary part of the index of refraction $k$ :

$$
K=\frac{4 \pi}{\lambda} k_{1}
$$

The attenuation of the amplitude of an incident wave by a film of thickness $h$ can further be written as:

$$
\hat{E}(h)=\hat{E}_{I} e^{-\frac{K}{2} h}
$$

Therefore, the reflected amplitudes $E_{R 0}$ and $E_{R 1}$ can be expressed with knowledge of the Fresnel coefficients and the absorption coefficient $K$ :

$$
\begin{aligned}
& \widehat{E}_{R 0}=\widehat{E}_{I}\left|r_{01}\right| \\
& \widehat{E}_{R 1}=\widehat{E}_{I}\left|r_{12} t_{01} t_{10}\right| e^{-\frac{K}{2} 2 h}
\end{aligned}
$$

In the cases of constructive and destructive interference these two waves now are added (i.e. both waves are in phase) and subtracted (phase shift of $\pi$ ) respectively:

$$
\begin{aligned}
& \widehat{E}_{R \text { Rons }}=\widehat{E}_{R 0}+\widehat{E}_{R 1}=\widehat{E}_{I}\left(\left|r_{01}\right|+\left|r_{12} t_{01} t_{10}\right| e^{-\frac{K}{2} 2 h}\right) \\
& \widehat{E}_{\text {Rdes }}=\widehat{E}_{R 0}-\widehat{E}_{R 1}=\widehat{E}_{I}\left(\left|r_{01}\right|-\left|r_{12} t_{01} t_{10}\right| e^{-\frac{K}{2} 2 h}\right)
\end{aligned}
$$

Taking into account that $\rho=|r|^{2}$ and $\varepsilon=1-\rho$, the envelope of the emissivity signal can finally be written as: 


$$
\begin{gathered}
\widehat{\varepsilon}_{\text {cons }}=1-\left(\left|r_{01}\right|^{2}+\left|r_{12} t_{01} t_{10}\right|^{2} e^{-K 2 h}+2\left|r_{01}\right|\left|r_{12} t_{01} t_{10}\right| e^{-\frac{K}{2} 2 h}\right) \\
\widehat{\varepsilon}_{\text {des }}=1-\left(\left|r_{01}\right|^{2}+\left|r_{12} t_{01} t_{10}\right|^{2} e^{-K 2 h}-2\left|r_{01}\right|\left|r_{12} t_{01} t_{10}\right| e^{-\frac{K}{2} 2 h}\right)
\end{gathered}
$$

The result is illustrated in Fig.2 (left). The solid line shows the resulting emissivity, taking into account changes of phase, while the dotted lines show the envelope signal according to equation (9).

\section{Determination of the optical constants $(n, k)$}

From equation (9) it can be deduced that the reflectance of the system approaches a horizontal asymptote with an absolute value of $1-\left|r_{01}\right|^{2}$ as the film-thickness increases. We now subtract this value from the reflectance signal (Fig.3) and the new envelope is given by:

$$
\begin{aligned}
& \hat{\rho}_{\text {cons }}-\left|r_{01}\right|^{2}=\left|r_{12} t_{01} t_{10}\right|^{2} e^{-K 2 h}+2\left|r_{01}\right|\left|r_{12} t_{01} t_{10}\right|^{-\frac{K}{2} 2 h} \\
& \hat{\rho}_{\text {des }}-\left|r_{01}\right|^{2}=\left|r_{12} t_{01} t_{10}\right|^{2} e^{-K 2 h}-2\left|r_{01}\right|\left|r_{12} t_{01} t_{10}\right| e^{-\frac{K}{2} 2 h}
\end{aligned}
$$

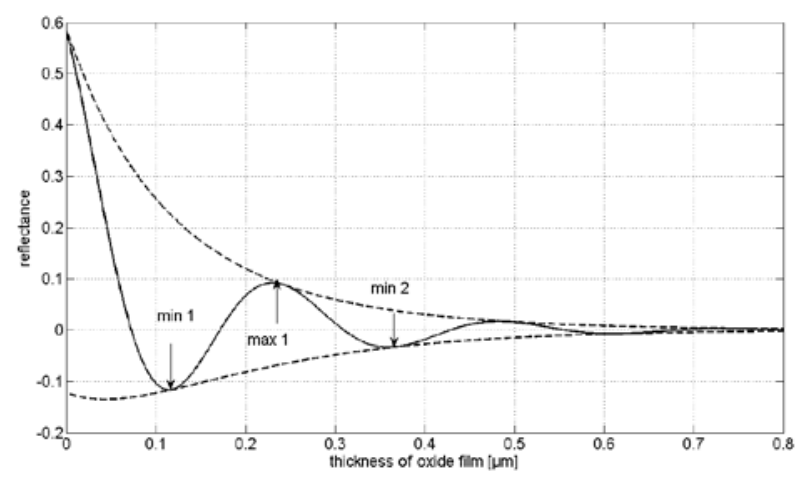

Fig.3: Reflectance and envelope (after subtraction of the final reflectivity $\left|r_{01}\right|^{2}$ ).

For the determination of the optical constants of the thin film, the absolute values of reflectance and film thickness (at positions min1, max1 and min2) have to be determined (see equation (13)). From the measured emissivity signal the absolute values of reflectance can be derived (as $\rho=1-\varepsilon$ ), but not the resulting film thickness. However, constructive and destructive interference appear periodically and can be related to film-thickness as follows:

$$
\begin{aligned}
h_{\text {des }} & =\frac{m \lambda}{2 n_{1}}+h_{0} \\
h_{\text {cons }} & =\frac{\left(m+\frac{1}{2}\right) \lambda}{2 n_{1}}+h_{0}
\end{aligned}
$$

with $m=0,1,2,3, \ldots$ and where $h_{0}$ is the thickness where the first destructive interference (min1) appears. The distance (i.e. absolute increase of film thickness) between successive minima and maxima only depends on the real part of the refractive index of the film $\left(n_{1}\right)$ as well as the wavelength of light and is given by: 


$$
\Delta h=\frac{\lambda}{4 n_{1}}
$$

From equations (11) and (12) the thickness at positions min1, max1 and min2 (as shown in Fig.3) can now be written as:

$$
\begin{aligned}
& h_{\min 1}=h_{0} \\
& h_{\max 1}=h_{0}+\frac{\lambda}{4 n_{1}} \\
& h_{\min 2}=h_{0}+\frac{\lambda}{2 n_{1}}
\end{aligned}
$$

These film thicknesses and the according measured reflectance values are inserted into the envelope equation (10). Thus three equations can be written, constraining the solution:

$$
\begin{aligned}
& \left|r_{12} t_{01} t_{10}\right|^{2} e^{-K 2 h_{0}}-2\left|r_{01} r_{12} t_{01} t_{10}\right| e^{-\frac{K}{2} 2 h_{0}}=\min 1 \\
& \left|r_{12} t_{01} t_{10}\right|^{2} e^{-K 2\left(h_{0}+\frac{\lambda}{4 n_{1}}\right)}+2\left|r_{01} r_{12} t_{01} t_{10}\right| e^{-\frac{K}{2} 2\left(h_{0}+\frac{\lambda}{4 n_{1}}\right)}=\max 1 \\
& \left|r_{12} t_{01} t_{10}\right|^{2} e^{-K 2\left(h_{0}+\frac{\lambda}{2 n_{1}}\right)}-2\left|r_{01} r_{12} t_{01} t_{10}\right| e^{-\frac{K}{2} 2\left(h_{0}+\frac{\lambda}{2 n_{1}}\right)}=\min 1
\end{aligned}
$$

Each of these three equations can be described as the sum of two exponential functions each multiplied by a constant factor. The unknown thickness $h_{0}$ depends on the refractive indices of the substrate and the film. If $h_{0}$ is set to 0 the above equations are only valid if the constant factors change accordingly. Thus, renaming these factors to $A$ and $B$, taking equation (5) into consideration and substituting $u=e^{-\frac{\pi \cdot k_{1}}{n_{1}}}$, equations (14) can be rewritten:

$$
\begin{aligned}
A-B & =\min 1 \\
A u^{2}+B u & =\max 1 \\
A u^{4}-B u^{2} & =\min 2
\end{aligned}
$$

As the absolute values of $\min 1, \max 1$ and $\min 2$ can be determined from emissivity measurements, equations (15) can be solved for $u$ (e.g. with the aid of an appropriate software package like Mathematica $\left.{ }^{\mathrm{TM}}\right)$. When the film finally reaches a certain thickness (i.e. the interference effect disappears) the value of the final reflectance $\left(\rho_{01}=\left|r_{01}\right|^{2}\right)$ can be directly determined from the measured emissivity (as indicated in Fig.2, left) but also calculated according to the following equation, with $\tilde{n}_{0}=1$ (air):

$$
\left|r_{01}{ }^{2}\right|=\rho_{01}=\left|\frac{\tilde{n}_{1}-\tilde{n}_{0}}{\tilde{n}_{1}+\tilde{n}_{0}}\right|^{2}=\frac{n_{1}^{2}-2 n_{1}+1+k_{1}^{2}}{n_{1}^{2}+2 n_{1}+1+k_{1}^{2}}
$$

Taking equation (16), the substitution $u=e^{-\frac{\pi \cdot k_{1}}{n_{1}}}$ and the result for $u$ of equations (15) into account, it is finally possible to find the values of $n_{1}$ and $k_{1}$, where only the real valued solutions are physically meaningful:

$$
\begin{gathered}
n_{1}=\left(-\pi^{2}-\pi^{2} \rho_{01} \pm \sqrt{4 \pi^{4} \rho_{01}-\pi^{2} \ln (u)^{2}+2 \pi^{2} \rho_{01} \ln (u)^{2}-\pi^{2} \rho_{01}{ }^{2} \ln (u)^{2}}\right) / \\
\left(-\pi^{2}+\pi^{2} \rho_{01}-\ln (u)^{2}+\rho_{01} \ln (u)^{2}\right) \\
u=e^{-\frac{\pi \cdot k_{1}}{n_{1}}} \Rightarrow k_{1}=-\frac{\ln (u) n_{1}}{\pi}
\end{gathered}
$$




\section{Experimental setup}

\section{Test apparatus}

For our experiments a special furnace was built that allowed us to heat circular shaped steel specimens (diameter: $40 \mathrm{~mm}$; height: $10 \mathrm{~mm}$; Fig.4 right) to maximal temperatures of approx. $600{ }^{\circ} \mathrm{C}$. The average roughnesses $\left(R_{a}\right)$ of the polished specimens were about $40 \mathrm{~nm}$. The furnace shown in Fig.4 consists of a sealed chamber with a vacuum pump attached. The specimens were placed on electrical heating elements (Fig.4, middle). The temperature of the heating element was measured by a thermocouple and a PID controller was used to guarantee constant absolute temperatures. A second thermocouple was attached directly to the specimen. To avoid early oxidation while heating, the chamber was evacuated ( $-30 \mathrm{mbar})$ and flooded with nitrogen gas to ensure an oxygen-reduced atmosphere. The thermal emission of the heated steel sample was observed with a 12-bit CCD-camera with an optical bandpass filter (1000 nm, FWHM = 10nm) mounted in front of the camera lens. The camera images were taken at intervals of approx. 5 seconds (integration time: 4 seconds) and saved automatically for further analysis. Three blind holes on the specimen surface were used as radiation reference (Fig.4, right). Assuming a certain ratio between diameter and depth, these holes can act as 'blackbodies'. The emissivity within these holes is very close to 1 and does not change during the oxidation process. Therefore the emissivity of the surface can simply be determined as the ratio between measured surface intensity and this radiation reference intensity, ensuring reliable emissivity values even under slightly varying temperature conditions during the oxidation process. The software allowed the definition of several regions of interest (ROIs) in the image where the mean intensity was measured and divided by the blackbody reference intensity (Fig.5) in video real time, visualizing the temporal evolution of emissivity at different spatial positions of the specimen surface.

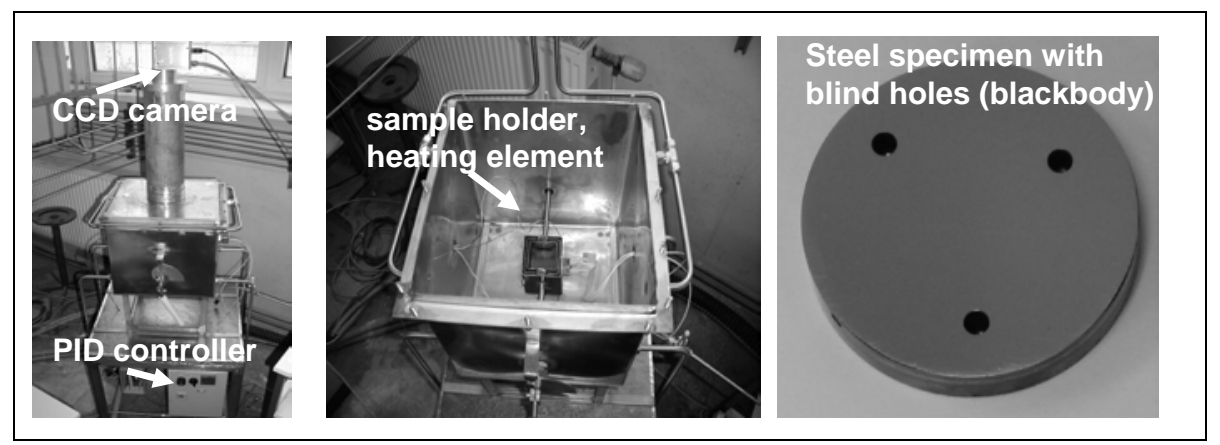

Fig.4: Left: furnace with CCD camera mounted with the image sensor parallel to the surface plane; midlle: sample holder and heating element; right: specimen with 3 blind holes acting as blackbody reference
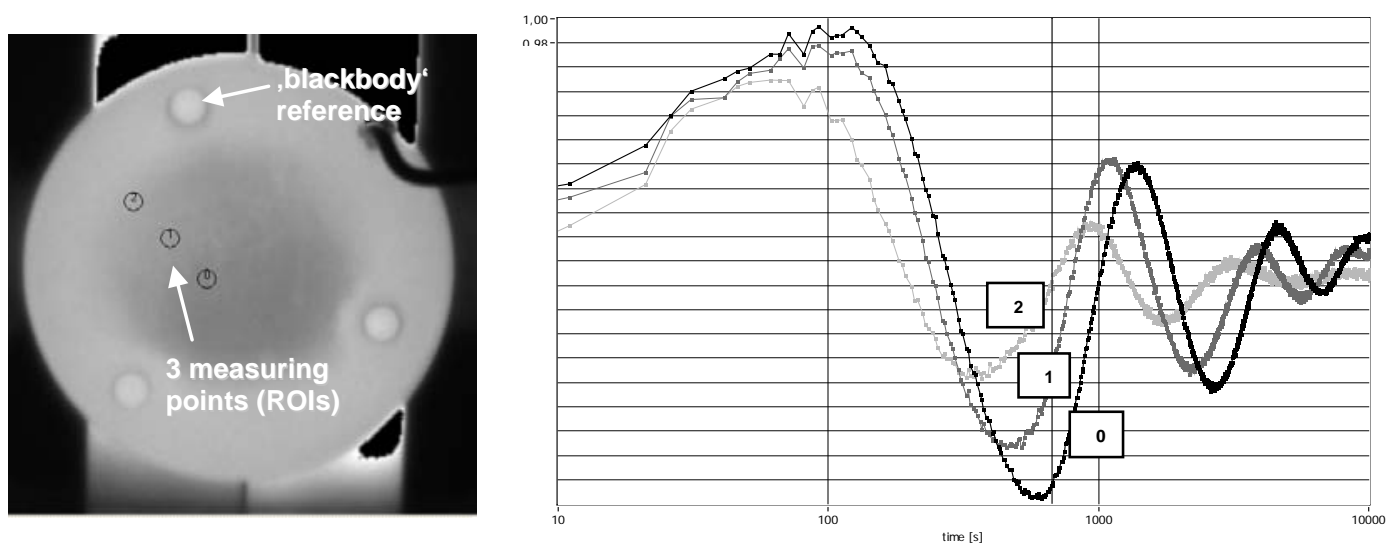

Fig.5: Left: CCD camera image of heated steel specimen; right: temporal emissivity signal at 3 different ROIs showing different film growth rates and optical properties at different surface positions 


\section{Results}

A resulting emissivity signal is shown exemplarily in Fig.6. Shown here are the emissivity values which were used for analysis (extreme values e1, e2, e3 and the final emissivity $\mathrm{e}_{\text {final }}$ ). It is worth mentioning, that any 3 consecutive extreme values can be used with the proposed method (exemplarily indicated by 'group1' and 'group2' in Fig.6, left). Thus, in addition to the analysis of the spatial distribution, the analysis of the temporal evolution of thin film optical properties is also possible (as shown in Fig.7). The values of the optical constants can be derived at each image pixel position by inserting the input parameters $\min 1, \max 1, \min 2$ and $\rho_{01}$ (according to Tab.1) into equations $(17-18)$. The results are in good agreement with ellipsometry results from literature (e.g. [7]).

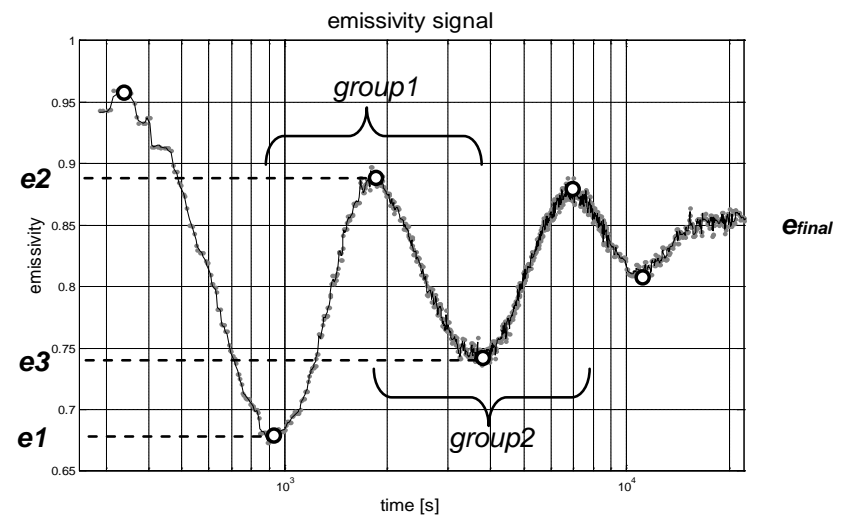

Table 1. Exemplarily calculated values of the optical constants based on the emissivity signal on the left hand side.

\begin{tabular}{|l|}
\hline $\boldsymbol{m i n} 1=(1-\mathrm{e} 1)-\left(1-\mathrm{e}_{\text {final }}\right)=(1-0.675)-(1-0.85)$ \\
\hline $\boldsymbol{m a x} 1=(1-\mathrm{e} 2)-\left(1-\mathrm{e}_{\text {final }}\right)=(1-0.891)-(1-0.85)$ \\
\hline $\boldsymbol{m i n} 2=(1-\mathrm{e} 3)-\left(1-\mathrm{e}_{\text {final }}\right)=(1-0.744)-(1-0.85)$ \\
$\rho_{01}=\left(1-\mathrm{e}_{\text {final }}\right)=(1-0.85)$ \\
\hline
\end{tabular}

$$
\boldsymbol{n}=2,25 \quad \boldsymbol{k}=0,13
$$

Fig.6: Analysis of a measured temporal emissivity signal at a certain image position

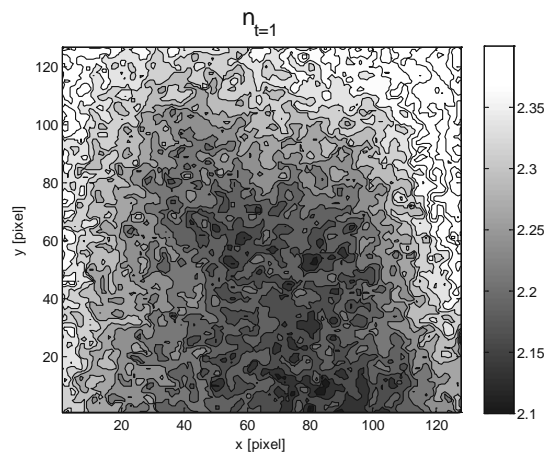

$\mathrm{k}_{\mathrm{t}=1}$

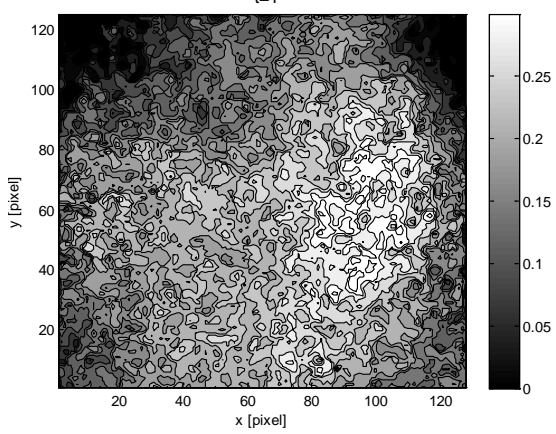

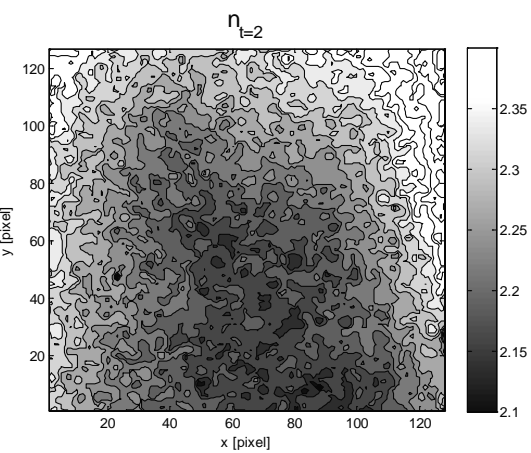

$\mathrm{k}_{\mathrm{t}=2}$

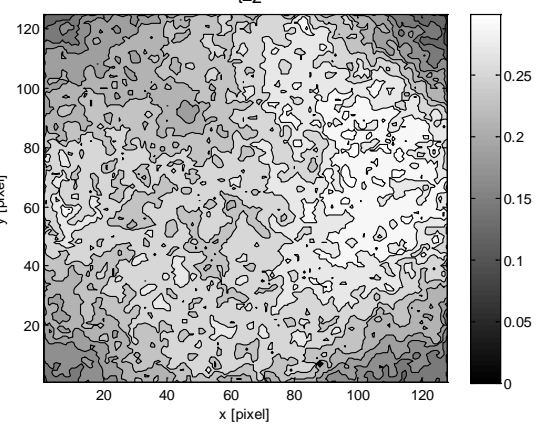

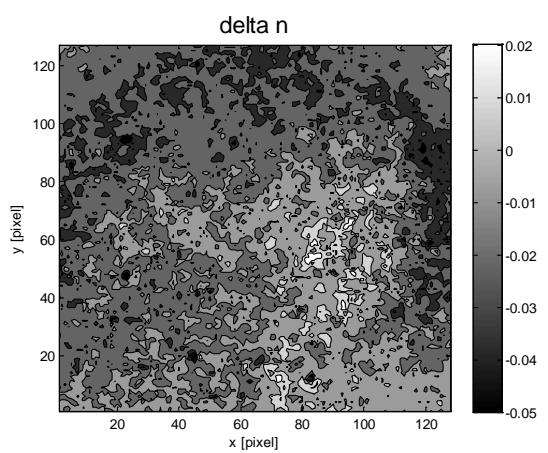

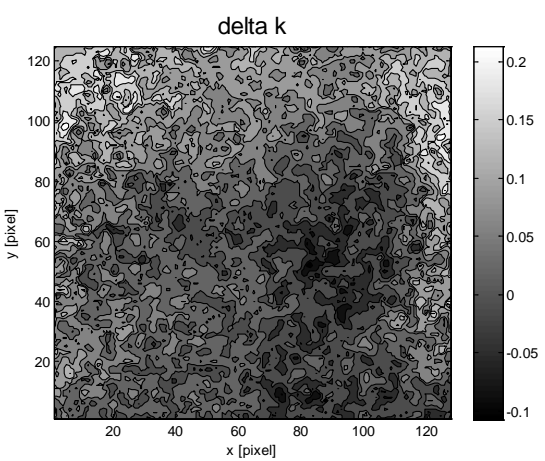

Fig.7: Images of the distribution of $n$ and $k$ values of an iron oxide layer (central part of the specimen) at two different instants of time (and the according difference, right column) 
The spatial distribution of $n, k$ - values of a typical specimen are shown in Fig.7. The left images show the results after analysis of 3 extreme values after approx. $3900 \mathrm{sec}$. of oxidation (group1) and the middle images accordingly after approx. $7000 \mathrm{sec}$. ('group2'). In each case the same value for $e_{\text {final }}\left(\rho_{01}\right)$ was assumed. Especially in the ' $n$ image' many of the image features reappear in the second image, showing the excellent reproducibility of the proposed approach (provided the surface properties do not change during film growth - in particular the final emissivity $e_{\text {final }}$ should be assumed as constant). The real part of the index of refraction $(n)$ shows distinct local variations which display a slight decrease towards the center of the specimen, whereas the absorption $(k)$ becomes more homogeneous during the oxidation process. A detailed analysis, especially concerning the theoretical accuracy when the input parameters are noisy or change significantly during the process (e.g. due to surface roughness effects), forms the basis of future work on this topic.

Based on the results for the real part of refraction $(n)$ the oxide layer thickness can now be calculated for every image pixel position according to equation (12), as every extreme value in the temporal emissivity signal indicates an increase of film thickness of $\lambda / 4 n$ (= approx. 100nm). Fig.8 shows the results obtained at two different oxidation temperatures (at approx. 30 mbar), showing quantitatively the temperature sensitivity of growth rates of thermally grown iron-oxide films.

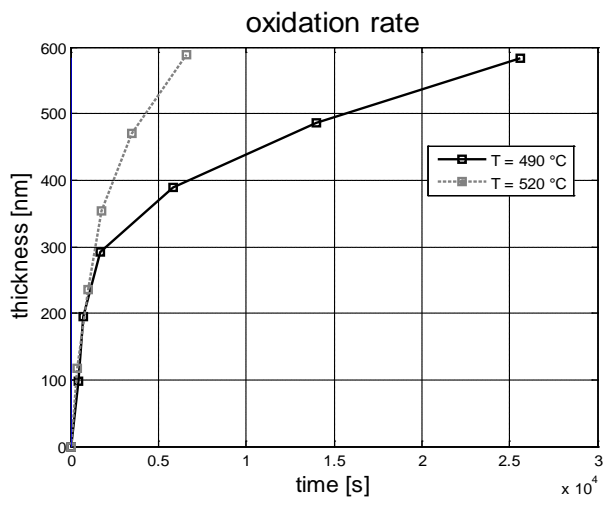

Fig.8: Measured oxidation rates for two different surface temperatures

\section{Summary}

A standard CCD camera is used for thermal imaging of heated steel specimens in the near infrared spectral range at temperatures above $400^{\circ} \mathrm{C}$. During the growth of a thin oxide layer the emissivity varies due to interference effects. In this work it is shown, that solely based on the analysis of four specific emissivity values (in particular three consecutive extreme values of the temporal emissivity signal and the final emissivity value, when interference effects disappear due to high absorption) the complex index of refraction of a thin film can be determined. The presented experimental setup allows a detailed study of thin film properties at high temperatures (e.g. growth rates or film inhomogeneities) spatially and temporally resolved. The proposed method is generally applicable to the analysis of optical properties of semitransparent absorbing thin films on absorbing substrates.

\section{REFERENCES}

[1] G. Zauner, et al. "CCD Cameras as Thermal Imaging Devices in Heat Treatment Processes", SPIE Proc. Electronic Imaging, San Jose, USA, 2004

[2] F. Maury, F.-D. Duminica "Diagnostics in TCOs CVD processes by IR pyrometry", Thin Solid Films, 2007

[3] P. Pigeat et al. "Calculation of thermal emissivity for thin films by a direct method", Physical Review B, Vol. 57/15, 1998

[4] T. Iuchi, T. Furukawa, S. Wada "Emissivity modeling of metals during the growth of oxide film and comparison of the model with experimental results", Applied Optics, Vol. 42, No. 13, 2003

[5] C. Gasqueres, F. Maury, F. Osola "In-Situ Optical Pyrometry in the CVD of Metallic Thin Films for Real Time Control of the Growth", Chem. Vap. Deposition, 2003, 9, No. 1

[6] O.S. Heavens "Optical Properties of Thin Solid Films", Butterworths Scientific Publications, London, 1955

[7] V. Goossens, et al. "Optical properties of thin iron oxide films on steel", Surface and Interface Analysis, 38:489-493, Wiley InterScience, 2006 